\title{
Autoimmune Hemolytic Anemia and Interstitial Pneumonitis as the Initial Presentation of Systemic Lupus Erythematosus
}

Sneha Phadke, D.O., Allan Fleming, M.D.

University of Kansas Medical Center, Department of Internal Medicine

\section{OBJECTIVE}

To emphasize the importance of considering hemolysis as a cause for anemia and the potentially catastrophic results that can occur if treatment is delayed.

\section{BACKGROUND}

- Autoimmune hemolytic anemia (AIHA) involves

immunologic destruction of erythrocytes and can be life-threatening.

- AIHA may be idiopathic, however, it should prompt a work-up for secondary causes.

- Evaluation consists of a reticulocyte count, LDH, haptoglobin, indirect bilirubin, direct and indirect Coombs tests, auto-antibody testing, and a peripheral smear for erythrocyte morphology, including microspherocytes, polychromasia, and schistocytes. - AIHA is not associated with a deficiency state; it is characterized by an elevated LDH and reticulocyte count, indirect hyperbilirubinemia, and a low haptoglobin.

Hemolytic Anemia

- Disseminated Intravascular Coagulation

- Thrombotic Thrombocytopenic Purpura

- Idiopathic and secondary antibody mediated

- Drug induced hemolytic anemia

Intrinsic Causes

- G-6-PD Deficiency

- Hereditary spherocytosis

- Hemoglobinopathies

\section{CASE REPORT}

- A 48-year-old female from Hong Kong with no significant past medical history presented to the emergency department with a one-month history of progressive dry cough and fatigue.

- On exam, she was noticeably icteric and had bibasilar coarse crackles.

- Labs on presentation: hemoglobin $4.9 \mathrm{~g} / \mathrm{dL}$, indirect bilirubin $7.1 \mathrm{mg} / \mathrm{dL}$, reticulocyte count 35\%, LDH $468 \mathrm{U} / \mathrm{L}$, haptoglobin $4 \mathrm{mg} / \mathrm{dL}$. Direct Coombs test positive secondary to warm agglutinins.

- Chest radiograph and CT scan revealed interstitial infiltrates.

- An immunologic work-up revealed positive anti-nuclear antibody, rheumatoid factor, anti-SSA, anti-Smith, anti-RNP, and a low complement level.

- High-dose intravenous corticosteroids were initiated, with a slow improvement in hemoglobin level as well as in the pulmonary infiltrates.

- Treatment has now been tapered to low dose oral corticosteroids, and the patient's hemoglobin remains within normal limits.

\section{RADIOLOGY}

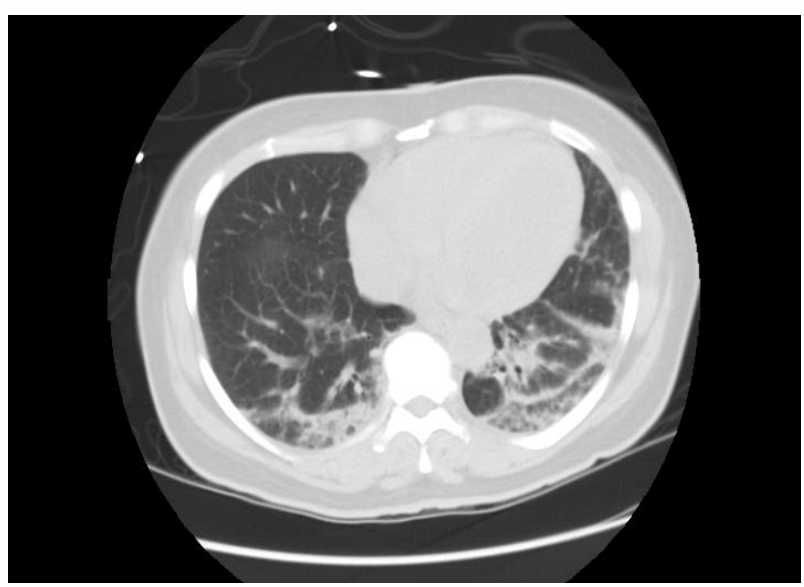

\section{DISCUSSION}

- The final diagnosis in this patient was systemic lupus erythematosus (SLE), presenting with autoimmune

hemolytic anemia and interstitial pneumonitis.

- In warm agglutinin mediated AIHA, IgG antibodies react at body temperature with protein antigens on the erythrocyte surface.

- This results in hemolysis by two mechanisms:

-(1) phagocytosis within the reticuloendothelial system, i.e. extravascular hemolysis, and

(2) complement-mediated erythrocyte destruction.

- Systemic corticosteroids are the mainstay of treatment,

while rarely, other immunosuppressants such as rituximab are used.

- Splenectomy may be necessary in refractory cases.

\begin{tabular}{|c|c|}
\hline$\overline{\text { Auto-antibody }}$ & Autoimmune Disease Associations \\
\hline Anti-SSA & Systemic Lupus Erythematosus (SLE), Sjogren's syndrome \\
\hline Anti-SSB & Sjogren's syndrome \\
\hline Anti-dsDNA & SLE \\
\hline Anti-centromere & CREST syndrome \\
\hline Anti-Smith & SLE \\
\hline Anti-histone & SLE and Drug Induced SLE \\
\hline Anti-topoisomerase & Systemic Sclerosis \\
\hline Rheumatoid Factor & SLE, Sjogren's, Rheumatoid Arthritis \\
\hline Anti-RNP & Mixed Connective Disease, SLF \\
\hline \multicolumn{2}{|c|}{ REFERENCES } \\
\hline 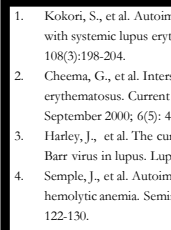 & 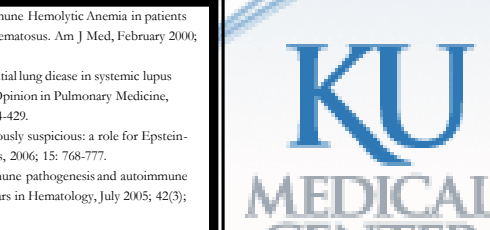 \\
\hline
\end{tabular}

